University of Wollongong

Research Online

Faculty of Social Sciences - Papers (Archive) Faculty of Arts, Social Sciences \& Humanities

2014

Exercise bra discomfort is associated with insufficient exercise levels among Australian women treated for breast cancer

Sheridan A. Gho

University of Wollongong, sgho@uow.edu.au

Bridget J. Munro

University of Wollongong, bmunro@uow.edu.au

Sandra C. Jones

University of Wollongong, sandraj@uow.edu.au

Julie R. Steele

University of Wollongong, jsteele@uow.edu.au

Follow this and additional works at: https://ro.uow.edu.au/sspapers

Part of the Education Commons, and the Social and Behavioral Sciences Commons

Research Online is the open access institutional repository for the University of Wollongong. For further information contact the UOW Library: research-pubs@uow.edu.au 


\title{
Exercise bra discomfort is associated with insufficient exercise levels among Australian women treated for breast cancer
}

\author{
Abstract \\ Purpose \\ Although participating in exercise is beneficial for breast cancer survivors, not being able to find a \\ comfortable exercise bra can be a barrier to exercise. It is likely that side effects specific to breast cancer \\ treatment exacerbate exercise bra discomfort. This study aimed to determine the relationship between \\ patient characteristics, physical side effects, exercise bra discomfort and exercise behaviours.
}

\section{Methods}

Four hundred thirty-two breast cancer survivors completed an online survey related to their treatment and demographic background, current exercise levels, reported exercise bra discomfort and breast cancer treatment side effects. Patient characteristics and exercise levels were considered in a binary logistic regression against reporting bra discomfort to ascertain significant relationships $(p<0.05)$ and predictive value (odds ratio). Pearson's chi-square statistics was used to determine significant relationships between reporting a side effect and exercise bra discomfort.

\section{Results}

Eight out of nine physical side effects were significantly related to reporting exercise bra discomfort. Reporting exercise bra discomfort was significantly related to not achieving a minimal recommended level of exercise.

\section{Conclusions}

This is the first study in the scientific literature that systematically links the reporting of exercise bra discomfort to not achieving recommended levels of exercise. This effect of bra discomfort on exercise was found after controlling for age, surgery type and current treatment among a large cohort of women treated for breast cancer. Furthermore, results from this study suggest that physical side effects, as a result of surgery and treatment associated with breast cancer, are linked to experiencing bra discomfort during exercise.

\section{Disciplines}

Education | Social and Behavioral Sciences

\section{Publication Details}

Gho, S. A., Munro, B. J., Jones, S. C. \& Steele, J. R. (2014). Exercise bra discomfort is associated with insufficient exercise levels among Australian women treated for breast cancer. Supportive Care in Cancer, 22 (3), 721-729. 
Manuscript Title: Exercise bra discomfort is associated with insufficient exercise levels among Australian women treated for breast cancer

\author{
Authors: Sheridan A. Gho ${ }^{1}$; Bridget J. Munro ${ }^{1,}$ Sandra C. Jones ${ }^{2}$; and Julie R. Steele ${ }^{1}$ \\ ${ }^{1}$ Biomechanics Research Laboratory, University of Wollongong, Wollongong, Australia \\ ${ }^{2}$ Centre for Health Initiatives, University of Wollongong, Wollongong, Australia
}

Corresponding author: Sheridan A. Gho, BSc (Hons), Biomechanics Research Laboratory, University of Wollongong, Wollongong, NSW 2522, Australia

Ph: (+61) 0242214480

Fax (+61) 0242215945

Email:sg490@uowmail.edu.au

Number of text pages: 10

Number of tables: 3

Number of figures: 1

Funding Source: This project was funded by the National Breast Cancer Foundation with the support of Cancer Australia. No other funding sources supported this work, and there are no financial disclosures from any of the authors.

October 2013 


\section{$\underline{\text { Abstract }}$}

Purpose: Although participating in exercise is beneficial for breast cancer survivors, not being able to find a comfortable exercise bra can be a barrier to exercise. It is likely that side effects specific to breast cancer treatment exacerbate exercise bra discomfort. This study aimed to determine the relationship between patient characteristics, physical side effects, exercise bra discomfort, and exercise behaviours.

Methods: 432 breast cancer survivors completed an online survey related to their treatment and demographic background; current exercise levels; reported exercise bra discomfort; and breast cancer treatment side effects. Patient characteristics and exercise levels were considered in a binary logistic regression against reporting bra discomfort to ascertain significant relationships $(p<0.05)$ and predictive value (odds ratio). Pearson's Chi-Square statistics were used to determine significant relationships between reporting a side effect and exercise bra discomfort.

Results: Eight out of nine physical side effects were significantly related to reporting exercise bra discomfort. Reporting exercise bra discomfort was significantly related to not achieving a minimal recommended level of exercise.

Conclusions: This is the first study in the scientific literature that systematically links the reporting of exercise bra discomfort to not achieving recommended levels of exercise. This effect of bra discomfort on exercise was found after controlling for age, surgery type and current treatment among a large cohort of women treated for breast cancer. Furthermore, results from this study suggest that physical side effects, as a result of surgery and treatment associated with breast cancer, are linked to experiencing bra discomfort during exercise. 


\section{Introduction}

Participating in exercise has repeatedly been shown to be highly beneficial for the health and well-being of women who are treated for breast cancer [1-5]. Current literature indicates, however, that perceived barriers to exercise are associated with a reduced ability of these women to achieve recommended exercise levels [6-9]. Therefore, to encourage exercise participation among breast cancer survivors, these barriers to exercise need to be identified and minimised or removed [3]. In a recent study investigating 19 potential barriers to exercise, a lack of discipline, procrastination, being fatigued by exercise, and not being able to find a comfortable bra to exercise in, were ranked as the top four barriers to exercise by women treated for breast cancer [10]. Of these 19 barriers, bra discomfort is the highest ranked barrier that can be modified through an external intervention, such as by providing exercise bras that suit the needs of breast cancer survivors.

Bra discomfort presents a unique challenge to women treated for breast cancer due to the significant physical changes to the breast and surrounding tissue as a result of breast cancer treatment. Despite this, only one other study could be located which investigated the impact of bra discomfort on exercise levels among breast cancer survivors [10]. In that study, a significant proportion of respondents $(70 \%)$ experienced exercise bra discomfort, although this was not related to weekly exercise levels or reported physical side effects. This previous work, however, was limited by a small sample size, and an investigation of the effect of exercise bra discomfort on exercise behaviour, with sufficient statistical power to control for confounding demographic and treatment variables, is warranted.

Targeting patients who would benefit the most is vital when planning health or lifestyle interventions. As such, a better understanding of the women more likely to report exercise bra discomfort is essential when identifying women most likely to benefit from interventions aimed at improving exercise bra design. Patient characteristics such as age, surgery type, or whether the respondent was undergoing current treatment, have previously provided moderate 
associative value when analysed with respect to potential side effects of breast cancer treatment [11]. We postulate that using these same patient characteristics to identify women who are more likely to experience exercise bra discomfort could be an informative first step in developing strategies to improve exercise bra comfort. These strategies may include improving bra design or educating women towards improving bra fit.

Given the impact exercise barriers can have on exercise levels [6-9], and that exercise bra discomfort has been reported as a potential barrier to exercise [10], the effect of exercise bra discomfort on exercise behaviour, while controlling for the potentially participant characteristics should be explored. In order to develop strategies aimed at improving exercise bra comfort for women treated for breast cancer, a clearer understanding of the women who experience bra discomfort the most, as well as the physical side effects related to this bra discomfort is needed. Therefore, the primary aim of this study was to determine the relationship between exercise bra discomfort, exercise behaviours and patient characteristics among a large cohort of women who had been treated for breast cancer. The secondary aim of this study was to assess the relationship between physical side effects of breast cancer treatment and exercise bra discomfort. We hypothesised that women who experience exercise bra discomfort do not achieve sufficient levels of exercise, after controlling for patient characteristics. We also hypothesised that experiencing exercise bra discomfort is related to experiencing physical side effects as a result of breast cancer treatment.

\section{$\underline{\text { Participants and Methods }}$}

\section{Participants and Survey Implementation}

Breast cancer patients who had a registered email address with the Breast Cancer Network Australia (BCNA) Survey and Research Group or the Cancer Councils of Victoria or Western Australia were invited by email to complete an internet-based survey. Of the 482 women who visited the initial URL, 432 completed the survey ( $89.6 \%$ completion rate). This surpasses the calculated $n=384$ required sample size (based on a conservative $50 \%$ probability of obtaining 
statistical significance, assuming 95\% confidence interval, and a 10\% margin of error), thus providing sufficient statistical power for the following analysis [13]. Participant informed consent was obtained and the University Human Research Ethics Committee approved all data collection procedures (HREC08/326). The survey used in the present study formed part of a larger body of work, and details of the development, validation and content of the online survey instrument have been published elsewhere [11]. Survey items specific to the aims of the present study are described below.

\section{Analytical Variables}

Participant Characteristics: Participant age was an open-ended response to "What is your date of birth?", calculated with respect to the survey submission date (providing age at time of survey completion). Following this calculation, participants were split into categories of being "Under 50 years old", or "50 years and over" for the purpose of the binary logistical regression. Participant surgery type was assessed by a closed-ended question for which the responses were either a lumpectomy or mastectomy of either the right or left breast. Responses were not mutually exclusive, permitting participants to indicate whether they had undergone surgery on both breasts, or had a lumpectomy, followed by a mastectomy. Participants were then grouped into categories of a "lumpectomy" or a "mastectomy" for the binary logistic regression.

Finally, participants were asked "Are you CURRENTLY undergoing any of the following treatments for your breast cancer?" with closed-ended, non-mutually exclusive, response categories of chemotherapy, radiotherapy and hormonal treatment. For the purpose of the binary logistic regression, participants were divided into categories of "currently undergoing treatment" versus "finished treatment". Women who were still taking any medication for their breast cancer were classified as "currently undergoing treatment". Women were also asked the date of their "last treatment ever" if they had finished treatment. The difference between the 
survey completion date and treatment completion date was calculated to give a time since treatment completion at the time of survey completion.

Side Effects: Physical side effects deemed to potentially have a direct effect on bra discomfort included lymphoedema, broken and painful ribs, weight gain, shoulder limitations, aching muscles, hot flushes, burning, sensitive skin or chafing, pain, and muscular chest wall pain. Participants were asked to rate their experience of each of these side effects on a 5-point Likert scale from none (1) to severe (5). For the purpose of binary logistic regressions, women were divided into categories of "No symptom experience" (Likert response $=1$ ) versus "Any level of experience" (Likert response $=2-5$ ).

Bra Discomfort: The exercise bra discomfort question was a direct, closed-ended response item which queried "Do parts of the bra you wear during exercise cause you discomfort?", to which participants responded either "Yes" or "No".

Exercise Levels: Recreational exercise levels were assessed using the Recreational Activities domain of the World Health Organisation's (WHO) Global Physical Activity Questionnaire Version 2 (GPAQ2) [14]. Based on GPAQ2 analysis guidelines, respondents were then classified into those who met the GPAQ2 threshold for achieving moderate or high levels of exercise and those who achieved low levels or no exercise. These classifications included any combination of moderate or vigorous intensity exercise resulting in $\geq 600$ MET-minutes a week; or $\geq 3$ days/week of vigorous intensity exercise for $\geq 20$ minutes a day; or $\geq 5$ days/week of moderate intensity exercise or walking for $\geq 30$ minutes a day, as per GPAQ2 guidelines. [14] Achieving moderate or vigorous exercise as identified by GPAQ2 is also equivalent to meeting the WHO's recommendation of 150 weekly minutes of moderate exercise, or 75 weekly minutes of vigorous exercise, or an equivalent combination of both [15]. For the purpose of the binary logistic regression analysis, women who achieved moderate or high levels of exercise were deemed as "sufficiently active" whereas women who achieved only low levels or no level of exercise were deemed as "insufficiently active". 


\section{Statistical Treatment}

Patient Characteristics, Exercise Levels and Exercise Bra Discomfort: Exercise bra discomfort was considered in a binary logistic regression against patient characteristics and exercise levels to ascertain any significant relationships. Whether a participant reported experiencing bra discomfort (none versus any level of discomfort) was inserted as a dependent variable against the independent variables of age ( $<50$ years versus $\geq 50$ years), type of surgery (lumpectomy versus mastectomy), whether women were currently undergoing treatment (current treatment versus finished treatment), and exercise levels (sufficiently active versus insufficiently active). This method of analysis has been previously employed in a cross-sectional survey data analysis with this population [11, 16], and ensures each independent variable is analysed while controlling for the other three independent variables.

Side Effects and Exercise Bra Discomfort: Whether a participant reported a side effect (none versus any) was analysed by a $2 \times 2$ cross-tabulation relative to whether the participant reported exercise bra discomfort in response to direct questioning (Yes/No). Relationship significance was assessed using a Pearson Chi-square test of independence $(p<0.05)$. All statistical analyses were completed using SPSS for Windows software (Version 17.0, SPSS Inc, Chicago, USA).

\section{$\underline{\text { Results }}$}

\section{Sample Overview}

Participants were 432 women who had been treated for breast cancer, aged between 23-77 years (mean $53.3 \pm 9.8$ years; see Table 1). Table 1 provides information on the participant's self-reported health status, location, age, and exercise levels with comparisons to Australian population data. The age spread of the sample was generally lower than the age of the wider Australian breast cancer population, which may skew results towards a younger breast cancer population. Despite this, the self-reported health status of the participants was similar to the general Australian female population; and the survey sample was spread across Australian 
States and Territories in similar proportions to the wider breast cancer population, with the exception of the Australian Capital Territory, which formed $10 \%$ of the sample and only $2 \%$ of the national spread. The proportion of women deemed sufficiently active in the survey sample was very comparable to an age-matched general Australian female population $(36.5 \%$ vs. $37.6 \%)$.

One hundred and eighty eight women had under gone a lumpectomy during treatment for their breast cancer, 241 women had undergone a mastectomy, and 3 women reported no surgery (43.5\%, 55.8\% and $0.7 \%$, respectively; see Table 2). None of the women had differing procedures on both breasts, and the mastectomy classification included women who had a lumpectomy followed by a mastectomy. Two hundred and thirty nine women were currently undergoing treatment (or still taking medication for their breast cancer), 148 women finished all treatment, and 45 women did not respond to the question $(55.3 \%$ and $34.3 \%$ and $10.4 \%$, respectively; see Table 2). Only 158 women (36.5\%) were considered sufficiently active, which is close to the percentage of sufficiently active women in an age-matched general Australian female population (37.6\%; see Table 1)[34]. Exercise data were missing for nine participants. In all categories, missing data were treated using listwise deletion, and although this resulted in data loss of up to $13 \%$, this approach was deemed appropriate to provide unbiased parameter estimates.

\section{Patient Characteristics, Exercise Levels and Exercise Bra Discomfort}

Among respondents aged 50 years and over, the proportion of women reporting bra discomfort (28\%) was lower than the proportion who reported no bra discomfort $(36 \%)$. The same trend was observed to a smaller extent among women under 50 years old $(15 \%$ reporting discomfort; $17 \%$ reporting no discomfort). Among both lumpectomy and mastectomy groups, the same trend was observed again, whereby the proportion of women reporting bra discomfort (18\% lumpectomy; $25 \%$ mastectomy) was lower than the proportions of women reporting no bra discomfort (24\% and $28 \%$ respectively). This trend was also observed in 
current treatment groups, whereby the proportion of women reporting bra discomfort $(25 \%$ current treatment; $14 \%$ finished all treatment) was lower than the proportions of women reporting no bra discomfort (29\% and $19 \%$ respectively). However, the trend was reversed among insufficiently active respondents, whereby the proportion of women reporting bra discomfort (31\%) was greater than the proportion who reported no bra discomfort (29\%).

Consistent with these sample proportion observations, none of the patient characteristics of age, undergoing current treatment, or surgery type was significantly related to whether a participant reported experiencing exercise bra discomfort (see Table 3). However, reporting exercise bra discomfort was significantly related to a participant being sufficiently active. Specifically, women who reported exercise bra discomfort were less likely to be sufficiently active, while controlling for age, time post treatment and surgery type (OR 2.04; 1.32 - 3.16 $95 \% \mathrm{CI} ; p=0.04)$.

\section{Side Effects and Exercise Bra Discomfort}

The most commonly reported physical side effects were hot flushes $(\mathrm{n}=257)$, aching muscles $(\mathrm{n}=242)$, shoulder limitations $(\mathrm{n}=190)$, and pain $(\mathrm{n}=185)$. Across all physical side effects except hot flushes, the proportion of women reporting exercise bra discomfort was greater than the proportion of women not reporting this discomfort (see Figure 1). Also with the exception of hot flushes, all of the physical side effects reported by the respondents were significantly related to exercise bra discomfort (Pearson Chi-Square statistic; $p<0.05$ ).

\section{$\underline{\text { Discussion }}$}

As exercise bra discomfort has been identified as a significant barrier to exercise for women treated for breast cancer [10], this study aimed to increase the body of knowledge in this sparse area of research by determining whether any relationships existed between patient characteristics, exercise levels, and exercise bra discomfort. In agreement with our first hypothesis, women who experienced exercise bra discomfort were more likely to not achieve sufficient levels of exercise, after controlling for patient characteristics. In agreement with 
our second hypothesis, results from this study suggest that the physical side effects experienced as a result of surgery and treatment associated with breast cancer are linked to experiencing bra discomfort during exercise. The implications of these unique findings are discussed below.

\section{Exercise Bra Discomfort and Exercise Levels}

To maintain overall health, the WHO recommends adults aged 18-64 years should do at least 150 minutes of moderate-intensity aerobic physical activity throughout the week; or do at least 75 minutes of vigorous-intensity aerobic physical activity throughout the week; or an equivalent combination of moderate- and vigorous-intensity activity [15]. Within this study, irrespective of age, current treatment or type of surgery, women who reported bra discomfort were significantly less likely to achieve the minimum recommended level of exercise. We postulate that this result implies that the level of bra discomfort experienced by a respondent was sufficient to impede that respondent's ability to achieve these recommended levels of exercise. This finding is of concern given the well-established benefits of exercise for this cohort, and the potential for this barrier to be alleviated or reduced by providing more effective and comfortable exercise bra designs, which are specific to the needs of breast cancer survivors. The specific exercise bra design needs of breast cancer survivors are likely linked to the side effects of their treatment, and so an investigation into the relationship between treatment side effects and exercise bra discomfort was also warranted. The present study found that, with the exception of hot flushes, all the reported physical side effects associated with respondents' breast cancer treatments were significantly related to the reporting of exercise bra discomfort.

\section{Side Effects and Bra Discomfort}

\section{Surgical Side Effects}

Surgery for breast cancer is associated with considerable short and long-term morbidity, which may include lymphoedema, shoulder limitations, and pain [17, 18]. Upper-body 
morbidity, which encompasses both lymphoedema and shoulder limitations, is a severe and chronic condition affecting $19-54 \%$ of breast cancer patients even up to 3 years post treatment [19]. Although outcomes are variable, the more extensive surgeries, such as axillary dissections and accompanying radiation- and chemo- therapies, are linked to the development of upper body morbidities. Many women who undergo breast surgery also suffer from illdefined pain syndromes [20]. Pain that is a direct consequence of surgery can be nociceptive (resulting from injury to ligament or muscle); or neuropathic (resulting from injury to the nerves innervating the region), and affects $20 \%$ to $75 \%$ of women following a mastectomy $[17,21]$. Nociceptive pain usually resolves as the damaged tissues heal, whereas neuropathic pain may develop into a chronic syndrome [21]. Of particular relevance to bra designs for women following surgery for breast cancer is the development of neuroma pain, a chronic neuropathic pain arising from peripheral nerves being severed or injured and entrapped within scar tissue. These scars can cause spontaneous pain and severe mechanosensitivity [21], which can be exacerbated by both breast motion and contact of the bra over the scar tissue.

The present study found reporting lymphoedema, shoulder limitations, aching muscles, pain, and muscular chest wall pain was significantly related to reporting exercise bra discomfort. Considering this link, and given that breast cancer surgery side effects are common and may persist for many years post surgery [35], there is clear need for further investigation into specialised breast support designs for women following surgery for breast cancer, who wish to reap the health benefits associated with exercise.

\section{Radiotherapy Side Effects}

The physical side effects of broken and painful ribs, as well as burning, sensitive skin and/or chafing, are typically linked to radiation therapy [17]. For the remainder of their lives, breast cancer survivors who have undergone radiation therapy are also at risk of developing longterm radiation effects such as lymphoedema, shoulder limitations and fibrosis [17]. Acute skin reactions due to radiation therapy are primarily due to a damaging effect on the basal layer of 
the epidermis [22]. Within three months of radiation, $61 \%$ of the patients report erythema, and $55 \%$ report pain and tenderness of the skin or breast [22]. Even six months after radiation, up to $44 \%$ of patients still experience pain and tenderness in the breast region, and up to $25 \%$ report erythema [22]. It has been suggested that radiation effects have the same frequency and intensity regardless of the type of surgery undergone, and acute and late radiation-related morbidities are independent adverse effects, without a mechanistic relationship [23]. Factors such as treatment technique, beam energy, bra cup size and dose variation across the target volume all have a significant effect on the acute skin reaction observed [24].

The axilla and inframammary fold are commonly the sites of the most severe skin injury following radiation therapy [25]. The band of an exercise bra provides the primary support for the breasts, and will sit on these sites, which may lead to greater discomfort as a result of bra band pressure on the skin and underlying hypodermis. Therefore, exercise bras for breast cancer survivors must account for and minimise the exacerbation of this radiation damage by minimising the bra band pressure experienced at these sites.

In some cases, an early skin reaction to radiation therapy can progress to a chronic injury. A common chronic skin condition following breast cancer radiation is fibrosis, which is characterised by an increase in "stiffness" or loss of compliance in the soft tissue [26, 27]. Fibrosis is typically permanent, and in skin, subcutis, and muscle, fibrosis can cause limitations in the range of motion and substantially affect function [26, 27]. This late effect of fibrosis has implications for exercise bra design for women following radiation therapy for breast cancer, as a "stiffer" breast may impair natural breast motion. An exploratory biomechanical study [28] found that, compared to the unaffected breast, the natural affected breast of four lumpectomy patients moved in an altered and restricted pattern when they ran on a treadmill, which was perceived by the participants as asymmetrical breast motion. We postulate that a prosthetic or reconstructed breast will also display motion changes, which may be detected by patients. These asymmetrical breast motion patterns are not accounted for 
in bras worn during exercise by breast cancer survivors, and thereby may result in exercise bra discomfort, or self-consciousness, which may be interpreted as feeling 'uncomfortable' in the bra. In fact, breast cancer survivors frequently report a fear of their prosthesis moving or falling out of their bra, which may be exacerbated by exercise, and excessive asymmetrical breast movement may draw unwanted attention to the survivor [29, 30]. Further study is therefore urgently required in order to ensure exercise bras designed for women treated for breast cancer account for the differences in movement likely to be displayed by the affected breast or prosthesis compared to the natural breast of these women.

The present study also found respondent weight gain was significantly linked to exercise bra discomfort. Weight gain is a common side effect of breast cancer treatment and carries with it an increased risk of secondary cancer, and the development of other morbidities [17, 31]. Weight gain is also associated with a change in body composition and an increase in body weight without concurrent increases in lean muscle mass (sarcopenic obesity) [17, 31]. Women who gain weight following breast cancer treatment often find asymmetrical gains between their affected and unaffected side, and this is even more poignant for women who use a prosthesis as a result of breast tissue removal [30]. As a result, fluctuations in weight will affect how balanced a survivor feels towards her unaffected side, and will also change the fit of a bra. Specifically, breast cancer survivors have reported difficulty matching the affected or unaffected breast, and/or prosthesis cup sizes within a bra [28]. Correct bra fit is imperative in order to achieve exercise bra comfort [36], which may explain why women treated for breast cancer who report weight gain also report exercise bra discomfort.

\section{Strengths and Limitations}

We acknowledge that the primary limitation of this study is that the exercise data are based on self-reported measures. Demographic data regarding marital status, income, education level, region or residence (regional/remote or metropolitan), as well as data regarding exercise behaviours prior to treatment for breast cancer, were not collected, and therefore could not be 
controlled for in the analysis of exercise behaviour. As this is the first study to investigate exercise bra discomfort and exercise levels among women living with breast cancer, the study was limited in the comparisons that could be made to existing literature specific to this field of research. Finally, we acknowledge that the coding of side effects into "no symptoms" versus "any level of that symptom" was broad. However, division into categories that accounted for the extent of symptom experience (none, mild to moderate, moderate to severe) was not feasible due to a limited distribution of respondents across the spectrum of symptom experience. Furthermore, even with this broad level of coding, the sub-sample sizes of these side effects were lower than what was deemed sufficient by a conservative proportional sample size calculation, and may limit the significance of these findings. Future studies with a larger study population, which can draw meaningful sub-sample sizes would be required to achieve this level of analysis. Future research should also gather greater details of which parts of the bra cause discomfort for women treated for breast cancer. Despite these limitations, this study provides valuable insight into an otherwise limited research area. The strengths of the study are that the online survey completion rate was very high $(89.6 \%)$, providing responses from a large sample of Australian women treated for breast cancer. Furthermore, the electronic nature of survey delivery allowed for side effect items to be randomised preventing any ordering bias; and limited the human error potential, which is present during manual paper-survey data transcriptions into electronic statistical packages.

\section{Conclusion}

This study links the reporting of exercise bra discomfort to not achieving recommended levels of exercise among women treated for breast cancer. This study also suggests that the physical side effects experienced as a result of breast cancer treatment are linked to experiencing bra discomfort during exercise. Based on these findings it is postulated that providing better bra designs, which are specific to the needs of breast cancer survivors, may eliminate or reduce one of the important barriers to exercise. This, in turn, will enhance exercise participation in 
this patient population and enable women treated for breast cancer to enjoy the health benefits associated with an active lifestyle.

\section{Acknowledgement}

The authors thank the National Breast Cancer Foundation, Cancer Australia and the Breast Cancer Network Australia for their ongoing support throughout this work.

\section{Conflict of Interest}

This project was funded by the National Breast Cancer Foundation with the support of Cancer Australia. No other funding sources supported this work, and there are no financial disclosures from any of the authors. The corresponding author (SG) has full control of all primary data and agrees to let the journal review data if requested. 
Table 1: Respondents' demographic information with comparisons to Australian population data.

\begin{tabular}{|c|c|c|c|}
\hline & \multicolumn{2}{|c|}{ Present Study } & \multirow[t]{2}{*}{ Comparison Data (\%) } \\
\hline & $\mathbf{N}$ & $\%$ & \\
\hline Health Status & 428 & & Australian General Female Population [32] \\
\hline \multicolumn{4}{|l|}{ In general, would you say your health is: } \\
\hline Excellent & 55 & $12.9 \%$ & \multirow{2}{*}{$57 \%$ [Excellent or Very Good] } \\
\hline Very good & 167 & $39.0 \%$ & \\
\hline Good & 150 & $35.0 \%$ & $29 \%$ [Good] \\
\hline Fair & 44 & $10.3 \%$ & \multirow{3}{*}{$14 \%$ [Fair or Poor] } \\
\hline Poor & 6 & $1.4 \%$ & \\
\hline Missing data & 4 & $0.9 \%$ & \\
\hline State or Territory & 428 & & Australian Breast Cancer Population [33] \\
\hline \multicolumn{4}{|l|}{ What is your postcode? } \\
\hline New South Wales & 114 & $26.6 \%$ & $34 \%$ \\
\hline Victoria & 119 & $27.8 \%$ & $25 \%$ \\
\hline Queensland & 65 & $15.2 \%$ & $18 \%$ \\
\hline Western Australia & 26 & $6.1 \%$ & $9 \%$ \\
\hline South Australia & 41 & $9.6 \%$ & $9 \%$ \\
\hline Tasmania & 15 & $3.5 \%$ & $2 \%$ \\
\hline Australian Capital Territory & 44 & $10.3 \%$ & $2 \%$ \\
\hline Northern Territory & 4 & $0.9 \%$ & $0 \%$ \\
\hline Missing data & 4 & $0.9 \%$ & \\
\hline Age & 432 & & Australian Breast Cancer Population [33] \\
\hline \multicolumn{4}{|l|}{ What is your date of birth? } \\
\hline$<30$ years old & 2 & $0.5 \%$ & $0 \%$ \\
\hline $30-49$ years old & 142 & $32.9 \%$ & $12 \%$ \\
\hline $50-69$ years old & 271 & $62.7 \%$ & $51 \%$ \\
\hline $70+$ years old & 17 & $3.9 \%$ & $37 \%$ \\
\hline Missing data & 0 & & \\
\hline Exercise & 423 & & Age-matched Australian Female Population [34] \\
\hline Sufficiently Active & 158 & $36.5 \%$ & $37.6 \%$ \\
\hline Missing data & 9 & $2.2 \%$ & \\
\hline
\end{tabular}


Table 2: Respondents' characteristics divided into binary logistic regression groups.

\begin{tabular}{lllc}
\hline Binary Logistic Regression Groups & $\mathrm{n}$ & $\%$ & Categories \\
\hline $\begin{array}{l}\text { Age } \\
\text { What is your date of birth? }\end{array}$ & 432 & & \\
$<30$ & 2 & $0.0 \%$ & "Under 50 years old" \\
$30-49$ & 142 & $33.0 \%$ & $\mathrm{n}=144 ; 33 \%$ \\
$50-69$ & 271 & $63.0 \%$ & "50 years and over" \\
$70+$ & 17 & $4.0 \%$ & $\mathrm{n}=288 ; 67 \%$ \\
\hline
\end{tabular}

Exercise

423

In a typical week, on how many days do you do [moderate-intensity or vigorous-intensity] sports, fitness or recreational activities? [Number of days per week]

How much time do you spend doing [moderate-intensity or vigorous-intensity] sports, fitness or recreation ON A TYPICAL DAY? [Hours][Minutes]

\begin{tabular}{|c|c|c|c|}
\hline Insufficiently Active & 265 & $61.3 \%$ & "Insufficiently Active" \\
\hline Sufficiently Active & 158 & $36.5 \%$ & "Sufficiently Active" \\
\hline Missing data & 9 & $2.2 \%$ & \\
\hline
\end{tabular}

Please indicate ALL the surgeries you have undergone for your breast cancer. If you have not undergone any surgery, please skip this question. [Lumpectomy][Mastectomy] with [Right][Left]

Unilateral lumpectomy

$179 \quad 41.4 \%$

Double lumpectomy

$9 \quad 2.1 \%$

Unilateral lumpectomy followed by mastectomy

$75 \quad 17.4 \%$

Double lumpectomy followed by mastectomy

$24 \quad 5.6 \%$

Unilateral mastectomy

$107 \quad 24.8 \%$

Double mastectomy

$35 \quad 8.1 \%$

Missing data 3 $0.7 \%$

\begin{tabular}{c}
\hline "Lumpectomy" \\
\hline $\mathrm{n}=188 ; 43.5 \%$ \\
\hline $\mathrm{n}=241 ; 55.8 \%$ \\
\hline
\end{tabular}

Time since completion of treatment 387

Are you CURRENTLY undergoing any of the following treatments? [Chemotherapy][Radiotherapy][Hormonal therapies] For the treatments you have FINISHED, what was the date of your last session ever? [Month][Year]

$\begin{array}{lll}\text { Current chemotherapy } & 14 & 3.2 \% \\ \text { Current radiotherapy } & 3 & 0.7 \% \\ \text { Current hormonal therapies } & 226 & 51.4 \% \\ <1 \text { year } & 20 & 4.6 \% \\ 1-2 \text { years } & 50 & 11.5 \% \\ 3-4 \text { years } & 23 & 5.3 \% \\ 5-7 \text { years } & 29 & 6.7 \% \\ 8-10 \text { years } & 9 & 2.1 \% \\ >10 \text { years } & 17 & 3.9 \%\end{array}$

Mean ( \pm S.D) years of treatment completion (excluding current Tx)

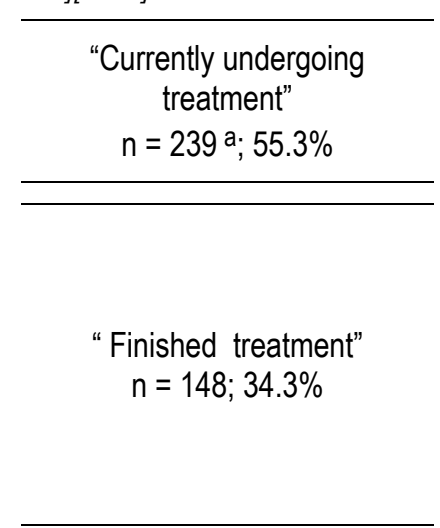

Missing data

45

$10.4 \%$

Two respondents were simultaneously undergoing chemotherapy and hormonal therapies, while another two respondents were undergoing radiotherapy and hormonal therapies. 
Table 3: Group percentage numbers and binary logistic regression values (with Odds Ratio) of patient characteristics and exercise levels against reported bra discomfort.

\begin{tabular}{|c|c|c|c|c|c|c|c|c|}
\hline \multirow[b]{2}{*}{ Bra discomfort a } & \multicolumn{8}{|c|}{ Independent Variables } \\
\hline & \multicolumn{2}{|c|}{$\begin{array}{c}\text { Age } \\
\text { Under } 50 \text { years old vs. } \\
50 \text { years and over }\end{array}$} & \multicolumn{2}{|c|}{$\begin{array}{c}\text { Surgery } \\
\text { Lumpectomy vs. } \\
\text { Mastectomy }\end{array}$} & \multicolumn{2}{|c|}{$\begin{array}{l}\text { Current Treatment } \\
\text { Current treatment vs. } \\
\text { Finished treatment }\end{array}$} & \multicolumn{2}{|c|}{$\begin{array}{c}\text { Exercise } \\
\text { Insufficiently active vs. } \\
\text { Sufficiently active }\end{array}$} \\
\hline \multirow[t]{2}{*}{ Odds Ratio $(95 \% \mathrm{Cl})^{\mathbf{b}}$} & \multicolumn{2}{|c|}{$1.01(0.65-1.58)$} & \multicolumn{2}{|c|}{$1.25(0.81-1.92)$} & \multicolumn{2}{|c|}{$0.88(0.58-1.35)$} & \multicolumn{2}{|c|}{$2.04^{*}(1.32-3.16)$} \\
\hline & $<50 \mathrm{yr}$ & $\geq 50 \mathrm{yr}$ & Lumpectomy & Mastectomy & $\begin{array}{l}\text { Current } \\
\text { treatment }\end{array}$ & $\begin{array}{l}\text { Finished } \\
\text { treatment }\end{array}$ & $\begin{array}{l}\text { Insufficiently } \\
\text { active }\end{array}$ & $\begin{array}{l}\text { Sufficiently } \\
\text { active }\end{array}$ \\
\hline $\begin{array}{l}\text { Number reporting bra } \\
\text { discomfort }(\%)\end{array}$ & $64(15 \%)$ & $120(28 \%)$ & $76(18 \%)$ & $107(25 \%)$ & $106(25 \%)$ & $59(14 \%)$ & $133(31 \%)$ & $51(12 \%)$ \\
\hline $\begin{array}{l}\text { Number reporting no } \\
\text { bra discomfort (\%) }\end{array}$ & $74(17 \%)$ & $156(36 \%)$ & $105(24 \%)$ & $123(28 \%)$ & $126(29 \%)$ & $82(19 \%)$ & $125(29 \%)$ & $105(24 \%)$ \\
\hline Data missing & \multicolumn{2}{|c|}{$18(4 \%)$} & \multicolumn{2}{|c|}{$21(5 \%)$} & \multicolumn{2}{|c|}{$59(13 \%)$} & \multicolumn{2}{|c|}{$18(4 \%)$} \\
\hline
\end{tabular}

a "Do parts of the bra you wear during exercise cause you discomfort?"; closed-ended response question, "Yes" or "No" response options.

b $O R=1$ : event equally likely in both groups; $O R>1$ : event more likely in first group; $O R<1$, event more likely in second group.

* $p=0.04$ 
Figure 1: Proportion of women who reported experiencing exercise bra discomfort and the associated physical side effects.

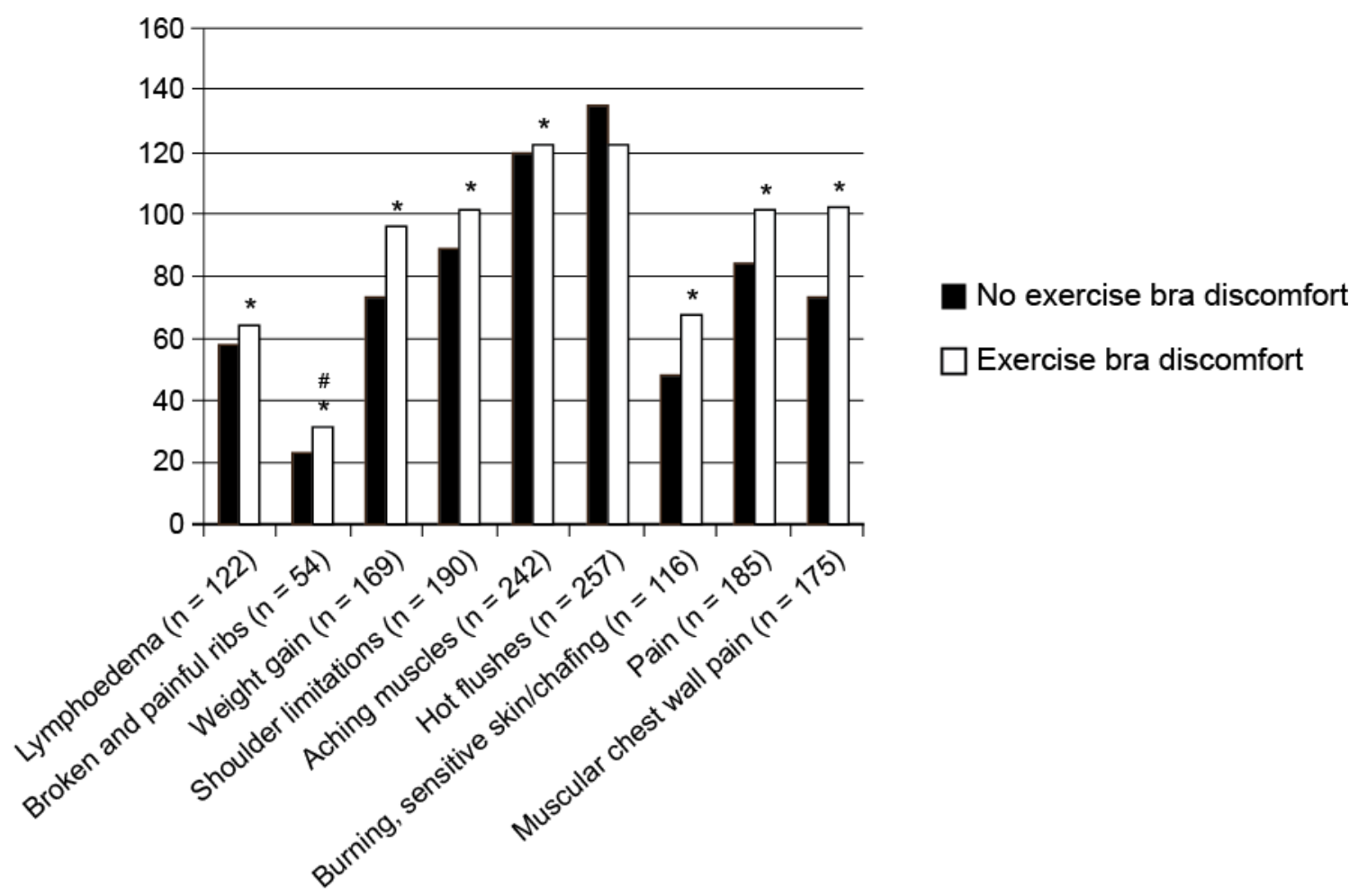




\section{References}

1. McNeely, M.L., et al., Effects of exercise on breast cancer patients and survivors: a systemic review and meta-analysis. Canadian Medical Association Journal, 2006. 175(1): p. 34-41.

2. Schmitz, K.H., et al., Controlled physical activity trials in cancer survivors: A systematic review and meta-analysis. Cancer Epidemiology Biomarkers \& Prevention, 2005. 14(7): p. 1588-1595.

3. Markes, M., T. Brockow, and K.L. Resch, Exercise for women receiving adjuvant therapy for breast cancer. Cochrane Database of Systematic Reviews, 2006(4).

4. McNeely, M.L., et al., Exercise interventions for upper-limb dysfunction due to breast cancer treatment. Cochrane Database of Systematic Reviews, 2010(6).

5. Speck, R., et al., An update of controlled physical activity trials in cancer survivors: a systematic review and meta-analysis. Journal of Cancer Survivorship, 2010. 4(2): p. 87-100.

6. $\quad$ Rogers, L.Q., et al., Reduced barriers mediated physical activity maintenance among breast cancer survivors. Journal of Sport and Exercise Psychology, 2011. 33(2): p. 235-254.

7. Perna, F.M., et al., Negative Affect and Barriers to Exercise Among Early Stage Breast Cancer Patients. Health Psychology, 2008. 27(2): p. 275-279.

8. Courneya, K.S., et al., Barriers to supervised exercise training in a randomized controlled trial of breast cancer patients receiving chemotherapy. Annals of Behavioral Medicine, 2008. 35(1): p. 116-22.

9. Courneya, K.S., et al., A longitudinal study of exercise barriers in colorectal cancer survivors participating in a randomized controlled trial. Annals of Behavioral Medicine, 2005. 29(2): p. 147-153.

10. Gho, S., J. Steele, and B. Munro, Is bra discomfort a barrier to exercise for breast cancer patients? Supportive Care in Cancer, 2010. 18(6): p. 735-741.

11. Gho, S.A., et al., Self- reported side effects of breast cancer treatment: A crosssectional study of incidence, predictors, and the influence of exercise. Cancer Causes and Control, 2012. 24: p. 517-528.

12. Lorenzten, D. and L. Lawson, Selected sports bras: a biomechanical analysis of breast motion while jogging. The Physician and SportsMedicine, 1987. 15(3): p. 128-30, 132134, 136, 139.

13. Aday, L.A., Designing and conducting health surveys. Second ed. 1996, San Francisco, California: Jossey-Bass Inc.

14. WHO. Global Physical Activity Questionaire (GPAQ). Department of Chronic Diseases and Health Promotion, Surveillance and Population-Based Prevention; Available from: http://www.who.int/chp/steps/resources/GPAQ_Analysis Guide.pdf.

15. WHO, Global recommendations on physical activity for health, World Health Organisation, Editor. 2010: Geneva.

16. Warmuth, M.A., et al., Complications of axillary lymph node dissection for carcinoma of the breast - A report based on a patient survey. Cancer, 1998. 83(7): p. 1362-1368.

17. Smoot, B., M. Wampler, and K.S. Topp, Breast cancer treatments and complications: Implications for rehabilitation. Rehabilitation Oncology, 2009. 27(3): p. 16-26.

18. Albert, U.S., et al., Early self-reported impairments in arm functioning of primary breast cancer patients predict late side effects of axillary lymph node dissection: results from a population-based cohort study. Breast Cancer Research \& Treatment, 2006. 100(3): p. 285-92.

19. Hayes, S.C., et al., Upper-body morbidity after breast cancer. Cancer, 2012. 118(S8): p. 2237-2249. 
20. Wallace, M.S., et al., Pain after breast surgery: a survey of 282 women. Pain, 1996. 66(2-3): p. 195-205.

21. Jung, B.F., et al., Neuropathic pain following breast cancer surgery: proposed classification and research update. Pain, 2003. 104(1-2): p. 1-13.

22. Holli, K., et al., Early skin and lung reactions in breast cancer patients after radiotherapy: prospective study. Radiotherapy and Oncology, 2002. 64(2): p. 163-169.

23. Lopez, E., et al., Early and late skin reactions to radiotherapy for breast cancer and their correlation with radiation-induced DNA damage in lymphocytes. Breast Cancer Research, 2005. 7: p. R690-R698.

24. Fernando, I.N., et al., An acute toxicity study on the effects of synchronous chemotherapy and radiotherapy in early stage breast cancer after conservative surgery. Clinical Oncology, 1996. 8(4): p. 234-238.

25. Harper, J.L., et al., Skin toxicity during breast irradiation: Pathophysiology and management. Southern Medical Journal, 2004. 97(10): p. 989-993.

26. Marinus, J., et al., Measuring radiation fibrosis: the interobserver reliability of two methods of determining the degree of radiation fibrosis. International Journal of Radiation Oncology*Biology*Physics, 2000. 47(5): p. 1209-1217.

27. Chopra, R.R. and J.A. Bogart, Radiation therapy-related toxicity (including pneumonitis and fibrosis). Hematology/Oncology Clinics of North America, 2010. 24(3): p. 625-642.

28. Gho, S.A., J.R. Steele, and B.J. Munro. Breast motion and vertical breast displacement in lumpectomy patients. in XXIIIrd Congress of the International Society of Biomechanics. 2011. Brussels, Belgium: International Society of Biomechanics.

29. Roberts, S., et al., External breast prosthesis use: Experiences and views of women with breast cancer, breast care nurses, and prosthesis fitters. Cancer nursing, 2003. 26(3): p. 179-186.

30. Gallagher, P., et al., External breast prostheses in post-mastectomy care: Women's qualitative accounts. European Journal of Cancer Care, 2010. 19(1): p. 61-71.

31. Demark-Wahnefried, W., et al., Changes in Weight, Body Composition, and Factors Influencing Energy Balance Among Premenopausal Breast Cancer Patients Receiving Adjuvant Chemotherapy. Journal of Clinical Oncology, 2001. 19(9): p. 2381-9.

32. AIHW, Australia's health. Australian Institute of Health and Welfare. Canberra: AIHW, 2010. Cat No. AUS 122.

33. AIHW, CA, and AACR, Cancer survival and prevalence in Australia: cancers diagnosed from 1982 to 2004. Australian Institute of Health and Welfare, Cancer Australia \& Australasian Association of Cancer Registries, 2008. Cancer Series No. 42: Cat. No: CAN 38.

34. ABS, Physical Activity in Australia: A Snapshot, 2007-08. 2011, Australian Bureau of Statistics.

35. Janz, N.K., et al., Symptom experience and quality of life of women following breast cancer treatment. J Women's Health 2007. 16:p.1348-1361

36. McGhee, D.E and J.R. Steele, Optimising breast support in female patients through correct bra fit. Journal of Science and Medicine in Sport, 2010. 13(6): p. 568-572 